\title{
The origin of the mass-metallicity relation: an analytical approach
}

\author{
E. Spitoni ${ }^{1}$, F. Calura ${ }^{2}$, F. Matteucci ${ }^{1,2}$, and S. Recchi ${ }^{3}$ \\ 1 Dipartimento di Fisica, Sezione di Astronomia, Università di Trieste, via G.B. Tiepolo 11, 34131, Italy \\ e-mail: spitoni@oats.inaf.it \\ 2 INAF Osservatorio Astronomico di Trieste, via G.B. Tiepolo 11, 34131, Italy \\ 3 Institute of Astronomy, Vienna University, Türkenschanzstrasse 17, 1180, Vienna, Austria
}

Received 3 December 2009 / Accepted 22 January 2010

\section{ABSTRACT}

\begin{abstract}
Context. The mass-metallicity (MZ) relation in star-forming galaxies at all redshifts has been recently established. It is therefore important to understand the physical mechanisms underlying such a relation.

Aims. We aim at studying some possible physical mechanisms contributing to the MZ relation by adopting analytical solutions of chemical evolution models, including infall and outflow.

Methods. Analytical models assume the instantaneous recycling approximation, which is still an acceptable assumption for elements produced on short timescales such as oxygen, which is the measured abundance in the MZ relation. We explore the hypotheses of a variable galactic wind rate, infall rate, and yield per stellar generation (i.e. a variation in the IMF), as possible causes for the MZ relation.

Results. By means of analytical models, we computed the expected $\mathrm{O}$ abundance for galaxies of a given total baryonic mass and gas mass. The stellar mass was derived observationally and the gas mass was derived by inverting the Kennicutt law of star formation, once its rate is known. Then we tested how the parameters describing the outflow, infall, and IMF should vary to reproduce the MZ relation, and we exclude the cases where such a variation leads to unrealistic situations.

Conclusions. We find that a galactic wind rate increasing with decreasing galactic mass or a variable IMF are both viable solutions for the MZ relation. A variable infall rate instead is not acceptable. It is difficult to differentiate among the outflow and IMF solutions by only considering the MZ relation, and other observational constraints should be taken into account in selecting a specific solution. For example, a variable efficiency of star formation increasing with galactic mass can also reproduce the MZ relation and explain the downsizing in star formation suggested for ellipticals. The best solution could be a variable efficiency of star formation coupled with galactic winds, which are indeed observed in low-mass galaxies.
\end{abstract}

Key words. ISM: abundances - Galaxy: abundances - Galaxy: stellar content - galaxies: evolution

\section{Introduction}

The study of the relation between the galactic stellar mass and the gas-phase metallicity, which represents the abundance of heavy elements present in the interstellar medium (hereinafter ISM), provides us with constraints on the various parameters fundamental for any galaxy formation theory. These parameters include the stellar initial mass function (IMF), representing the distribution of stellar masses at birth, which strongly influences the amount of heavy elements produced and restored by stars into the ISM. The mass-metallicity (hereinafter MZ) relation can also provide information on the star formation history: in general, a higher star formation rate (SFR) produces a higher concentration of metals in the ISM. Finally, the MZ relation provides information on the role of infall/outflow in galaxy evolution, namely on the amount of matter either ejected from galaxies into the intergalactic medium (IGM) or accreted onto galaxies from the IGM. Galactic outflows are directly connected to the importance of feedback and energy exchange between stars and interstellar gas.

At the present time, different theoretical explanations of the MZ relation have been proposed by various authors. The first explanation is based on starburst-induced galactic outflows, which are more efficient in expelling metal-enriched matter from lowmass galaxies than from giant galaxies, mainly owing to the shallower gravitational potential wells of the former (Larson 1974; Dekel \& Silk 1986; Tremonti et al. 2004; De Lucia et al. 2004; Kobayashi et al. 2007; Finlator \& Davé 2008). However, at the present time it is difficult to assess how the outflow efficiency depends on the baryonic galactic mass. In an alternative scenario, infall of pristine gas can dilute the interstellar metals and act in the same way as outflows, once one assumes longer infall timescales in lower mass galaxies (Dalcanton et al. 2004; but see also Dalcanton 2007).

Another explanation is that dwarf galaxies are less evolved than large galaxies, namely that the efficiency of star formation is greater in more massive systems. In this picture, large galaxies have formed the bulk of their stars by means of an intense star formation event at high redshift, quickly enriching their ISM to solar or over-solar metallicities, whereas dwarf galaxies, characterized by lower star formation efficiencies (i.e. SFRs per unit mass of gas) have subsolar interstellar metallicities. This interpretation is supported by various chemical evolution studies (Lequeux et al. 1979; Matteucci 1994; Calura et al. 2009), by cosmological $N$-body simulations (Brooks et al. 2007; Mouhcine et al. 2008; Tassis et al. 2008), and by hydrodynamical simulations (Tissera et al. 2005; De Rossi et al. 2007). A fourth way to produce an MZ relation is by means of a variable IMF as investigated in Köppen et al. (2007). It is possible that 
the relative importance of these processes may vary as a function of the total galactic stellar mass. However, the theoretical investigations performed so far do not allow us to disentagle these phenomena and to assess which may be considered dominant in galaxies of different masses.

In this paper, we aim at investigating the importance of different physical processes in determining the MZ relation by means of analytical chemical evolution models. This analytical approach will allow us to rapidly test the parameter space involved in our study, and it is justified because the oxygen evolution can be computed under the instantaneous recycling approximation, as is required for analytical solutions. By means of the solutions of the analytical models, including infall and outflow, we can compute the expected metallicity for a galaxy of a given mass and a given gas mass fraction. The total stellar mass and gas mass are derived from the observational data; in particular, we use the local MZ relation determined in SDSS galaxies, and from the SFRs observed in the same galaxy sample, we derive the gas mass fractions by adopting the Kennicutt (1998) law for star formation. We aim at assessing the importance of outflow, infall, and IMF (through the yield per stellar generation) in determining the $\mathrm{MZ}$ relation for galaxies of various masses. We also prove detailed expressions for the effective yields in different physical conditions, which can be used to compare the empirical effective yields as derived from observations (see Dalcanton 2007).

Our paper is organized as follows. In Sect. 2, we present our equations, our basic assumptions and the observables used in our study. In Sect. 3, we present our results, and finally, some conclusions are drawn in Sect. 4.

\section{Summary of simple-model solutions}

\subsection{The simple model}

We recall here the main assumptions and results of the simple models of chemical evolution (Tinsley 1980; Matteucci 2001; Recchi et al. 2008). As well known, the so-called Simple Model of chemical evolution is based on the following assumptions.

1. the system is one-zone and closed, namely there are no inflows or outflows;

2. the initial gas is primordial (no metals);

3. the IMF is constant in time;

4. the gas is well mixed at any time (instantaneous mixing approximation);

5. stars more massive than $1 M_{\odot}$ die instantaneously; stars smaller than $1 M_{\odot}$ live forever (instantaneous recycling approximation or IRA).

These simplifying assumptions allow us to calculate analytically the chemical evolution of the galaxies. We have defined the fundamental quantities, such as the returned fraction:

$R=\int_{1}^{\infty}\left(m-M_{R}\right) \phi(m) \mathrm{d} m$

where $\phi(m)$ is the IMF and $M_{R}$ is the mass of the remnant, and the yield per stellar generation:

$y_{Z}=\frac{1}{1-R} \int_{1}^{\infty} m p_{Z, m} \phi(m) \mathrm{d} m$,

where $p_{Z, m}$ is the fraction of newly produced and ejected metals by a star of mass $m$.
The well known solution of the closed box model can be easily found as:

$Z=y_{Z} \ln \left(\mu^{-1}\right)$

where $\mu$ is the gas fraction $M_{\mathrm{gas}} / M_{\mathrm{tot}}$, with $M_{\mathrm{tot}}=M_{*}+M_{\mathrm{gas}}$. This result is obtained by assuming that the galaxy initially contains only gas and has the remarkable property that it does not depend on the particular star formation history the galaxy experiences. Moreover, to obtain Eq. (3), one has to assume that $y_{Z}$ is constant in time. The yield that appears in Eq. (3) is known as effective yield, $y_{Z_{\text {eff }}}$, namely the yield the system would have if described by the simple model. In models with gas flows, the true yield as defined in Eq. (2) does not coincide with the effective yield, as we see in the next paragraphs.

\subsection{Leaky-box models}

Relaxing the first of the assumptions of the simple model, we get the models including gas flows, also known as leaky box models. Analytical solutions of simple models of chemical evolution including infall or outflow have been known for at least 30 years (Pagel \& Patchett 1975; Hartwick 1976; Twarog 1980; Edmunds 1990). Here we follow the approach and the terminology of Matteucci (2001); namely, for simplicity we assume linear flows (gas flows proportional to the SFR). Therefore, the outflow rate $W(t)$ is defined as

$W(t)=\lambda(1-R) \psi(t)$,

where $\psi(t)$ is the SFR, and the infall rate $A(t)$ is given by

$A(t)=\Lambda(1-R) \psi(t)$.

Here $\lambda$ and $\Lambda$ are two proportionality constants $\geq 0$. The first assumption is justified because, the greater the SFR, the more intense the energetic events associated with it (in particular supernova explosions and stellar winds), and therefore the larger the chance of having a large-scale outflow (see e.g. Silk 2003). A proportionality between $A(t)$ and $\psi(t)$ is less easily justified physically.

If we consider a system including outflow and infall, the set of equations we need to solve is the following one:

$\left\{\begin{array}{l}\frac{\mathrm{d} M_{\text {tot }}}{\mathrm{d} t}=(\Lambda-\lambda)(1-R) \psi(t) \\ \frac{\mathrm{d} M_{\mathrm{gas}}}{\mathrm{d} t}=(\Lambda-\lambda-1)(1-R) \psi(t) \\ \frac{\mathrm{d} M_{Z}}{\mathrm{~d} t}=(1-R) \psi(t)\left[\Lambda Z_{A}+y_{Z}-(\lambda+1) Z\right]\end{array}\right.$

where $M_{Z}$ is the mass of metals $\left(M_{Z}=Z \cdot M_{\text {gas }}\right)$ and $Z_{A}$ is the metallicity of the infalling gas.

\subsubsection{Simple model with outflow}

If we study a simple model with only outflow, e.g. $A(t)=0$, the system of Eq. (6) can be worked out, yielding the following solution (Matteucci 2001):

$Z=\frac{y_{Z}}{(1+\lambda)} \ln \left[(1+\lambda) \mu^{-1}-\lambda\right]$

For the integration we assume that at $t=0, Z(0)=0, M_{\mathrm{tot}}(0)=$ $M_{\text {gas }}(0)=M_{g, 0}$. Since $\mu$ values span the range $0 \leq \mu \leq 1$, the solution (7) is well-defined for all the values of $\lambda \geq 0$. In this case, the true yield is given by

$y_{Z}=\frac{Z(1+\lambda)}{\ln \left[(1+\lambda) \mu^{-1}-\lambda\right]}$ 
and it is clearly lower than the effective yield defined in Eq. (3). This should be kept in mind when using Eq. (3) to interpret observational data.

\subsubsection{Simple model with infall}

Here we analyze the opposite case: $A(t) \neq 0$ and $W(t)=$ 0 . Assuming for the infalling gas a primordial composition (e.g. $\left.Z_{A}=0\right), \Lambda \neq 1$ and $\Lambda \neq 0$, we obtain this solution (Matteucci 2001):

$Z=\frac{y_{Z}}{\Lambda}\left\{1-\left[\left(\Lambda-(\Lambda-1) \mu^{-1}\right]^{\frac{\Lambda}{\Lambda-1}}\right\}\right.$

We notice here that, in models with infall, not all the values of $\mu$ are allowed.If $\Lambda>1, \mu$ ranges between 1 and a minimum value:

$\mu_{\min }=\frac{\Lambda-1}{\Lambda}$.

For $\Lambda<1$, there is no $\mu_{\text {min }}$. If $\Lambda=1$ the solution is $Z=$ $y_{z}\left[1-\mathrm{e}^{-\left(\mu^{-1}-1\right)}\right]$, which is a well known solution of the extreme infall (Larson 1972), where the amount of gas remains constant in time.

\subsubsection{Simple model with inflow and outflow}

The general solution for a system described by the simple model in the presence of infall of gas with a general metallicity $Z_{A}$ and outflow is (Recchi et al. 2008)

$Z=\frac{\Lambda Z_{A}+y_{Z}}{\Lambda}\left\{1-\left[(\Lambda-\lambda)-(\Lambda-\lambda-1) \mu^{-1}\right]^{\frac{\Lambda}{\Lambda-\lambda-1}}\right\}$.

If the outflow rate is higher than the infall rate, the solution is defined for all $\mu$ values, not all values of $\mu$ are allowed in the opposite case.

In the models in which $\Lambda>\lambda+1, M_{\text {gas }}$ always increases (Eq. (6)), therefore $\mu$ ranges between 1 and a minimum value:

$\mu_{\min }=\frac{\Lambda-\lambda-1}{\Lambda-\lambda}$.

For models in which $\lambda+1>\Lambda>\lambda$, there is no $\mu_{\min }$, but there is an upper limit reachable by the gas mass, which is given by $M_{\mathrm{gas}, \lim }=M_{\mathrm{g}, 0} /(\lambda+1-\Lambda)$.

\subsubsection{Simple model with differential winds}

Recchi et al. (2008) present a set of new solutions in the simple model context and in the presence of differential winds, namely models where the metals are more easily channelled out of the parent galaxy than the pristine gas. The easiest way to consider a differential wind in the framework of simple models of chemical evolution is to assume that the metallicity of the gas carried out in the galactic wind is proportional to the metallicity of the ISM with a proportionality constant greater than one. If we define $Z^{\circ}$ as the metallicity of the outflowing gas, this condition implies that $Z^{\circ}=\beta Z$ with the ejection efficiency $\beta>1$. In the metallicity budget (third equation in (6)), we assume that the negative term due to the galactic wind is given by $W(t) Z^{\circ}=\beta \lambda(1-R) \psi(t)$.

With their simple approach, Recchi et al. (2008) are able to determine analytical expressions for the evolution of $Z$, which allow us to understand the effect of galactic winds on the chemical evolution of galaxies more clearly.

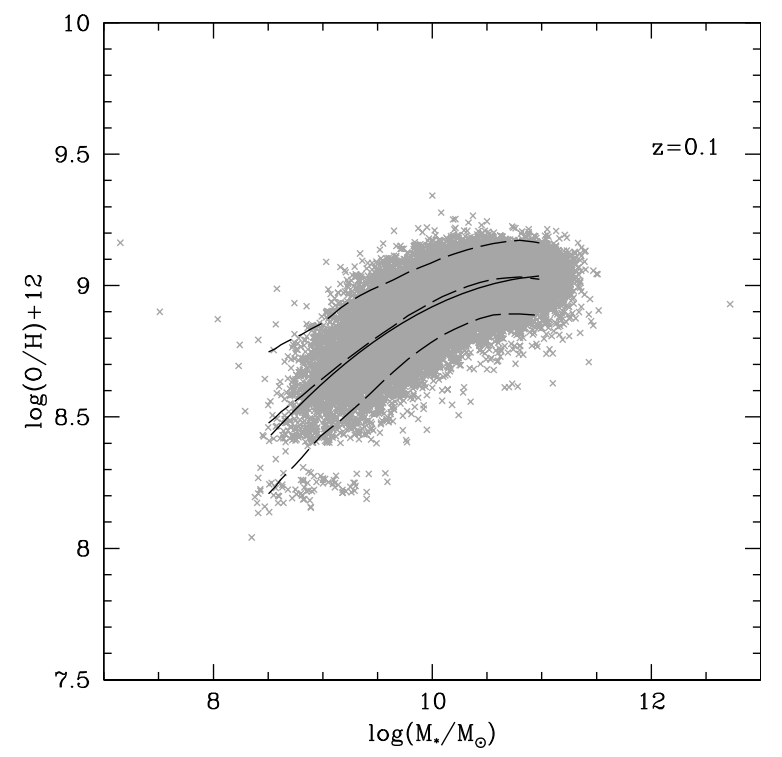

Fig. 1. The observed MZ relation for the oxygen (Kewley \& Ellison 2008). The dashed lines are the average values and the standard deviation and the solid one is the best fit to the average values as given by Maiolino et al. (2008). The redshift of the galaxies is indicated in the top right of the figure.

The set of the equations we solve in this case is very similar to (6), with the only difference given by the metallicity budget equation, which we modify as

$\frac{\mathrm{d} M_{Z}}{\mathrm{~d} t}=(1-R) \psi(t)\left[\Lambda Z_{A}+y_{Z}-(\lambda \beta+1) Z\right]$.

The solution of this new set of equations is given by

$Z=\frac{\Lambda Z_{A}+y_{Z}}{\Lambda+(\beta-1) \lambda}\left\{1-\left[(\Lambda-\lambda)-(\Lambda-\lambda-1) \mu^{-1}\right]^{\frac{\Lambda+(\beta-1) \lambda}{\Lambda-\lambda-1}}\right\}$

It is trivial to see that we can obtain Eq. (11) in the case $\beta=1$ (i.e. where the galactic wind is not differential).

\section{The observed mass-metallicity relation and our method}

Kewley \& Ellison (2008) analyzed the metallicity relation for Sloan Digit Sky Survey (SDSS) 27730 star-forming galaxies by adopting 10 different metallicity calibrations. In Fig. 1 we show the $\mathrm{MZ}$ relation at $z=0.1$ obtained by using the calibration of Kewley \& Dopita (2002), considered the best in the analysis by Calura et al. (2009), including the average values and the standard deviation.

In the same figure we also report the analytical fit of the observed MZ relation of Kewley \& Ellison (2008), as given by Maiolino et al. (2008), in particular,

$\log (\mathrm{O} / \mathrm{H})+12=-0.0864 *\left[\log \left(\frac{M_{*}}{M_{\odot}}\right)-11.18\right]^{2}+9.04$

Since from now on we use this analylitical fit as our fiducial MZ relation. For estimating the amount of gas that resides in each star-forming galaxy, we use the method described in Calura et al. (2008). We determine the cold gas mass of each galaxy on the basis of its SFR, by inverting the Kennicutt (1998) (hereafter K98) relation, which links the gas surface density to the SFR per unit area. A similar technique was used by Erb et al. (2006) 
and Erb (2008) to derive the gas fractions for a sample of starforming galaxies at $z \sim 2$ and to study the implications of the MZ relation observed at high redshift on the galactic gas accretion history, respectively.

Following K98, for any galaxy, the gas surface density $\Sigma_{\text {gas }}$, expressed in $M_{\odot} \mathrm{pc}^{-2}$, depends on the SFR surface density $\dot{\Sigma}_{*}$, expressed in $M_{\odot} \mathrm{yr}^{-1} \mathrm{kpc}^{-2}$ according

$\Sigma_{\text {gas }}=\left(\frac{\dot{\Sigma}_{*}}{2.5 \times 10^{-4}}\right)^{0.714} M_{\odot} \mathrm{pc}^{-2}$.

The scaling radius $R_{\mathrm{d}}$, which will be used to compute the SFR surface density profile of each galaxy, can be calculated as (Mo et al. 1998)

$R_{\mathrm{d}}=\frac{\lambda_{\mathrm{s}} R_{200} f_{c_{\mathrm{vir}}}^{-1 / 2} f_{R}\left(\lambda_{\mathrm{s}}, c_{\mathrm{vir}}, f_{\mathrm{b}}\right)}{\sqrt{2}}$

where $\lambda_{\mathrm{s}}$ is the spin parameter of the halo and depends on the total energy of the halo $E$, its angular momentum $J$ and its mass $M$ according to

$\lambda_{\mathrm{S}}=J|E|^{1 / 2} G^{-1} M^{-5 / 2}$.

The quantity $\lambda_{\mathrm{s}}$ is likely to assume values in the range $0.01 \leq$ $\lambda \leq 0.1$ (Heavens \& Peacock 1988; Barnes \& Efstathiou 1987; Jimenez et al. 1998). In this paper, we assume a typical value of $\lambda_{\mathrm{s}}=0.05$. Scatter in $\lambda_{\mathrm{s}}$ would propagate as scatter in the gas fractions, but we average over many galaxies to obtain the average gas fractions.

The parameter $c_{\mathrm{vir}}$ is the halo concentration factor, and it is calculated following Bullock et al. (2001) and Somerville et al. (2006), i.e., by defining a collapse redshift $z_{\mathrm{c}}$ for each halo, as $M_{*}\left(z_{\mathrm{c}}\right)=F \cdot M$. The value of $c_{\mathrm{vir}}$ is given by $c_{\mathrm{vir}}(M, z)=$ $K\left(1+z_{\mathrm{c}}\right) /(1+z)$, where $F$ and $K$ are two adjustable parameters. Following Somerville et al. (2006), we assume $K=3.4$ and $F=0.01$. Here we assume $z \sim 0.1$, corresponding to the average redshift of the SDSS galaxies (Tremonti et al. 2004).

To compute the quantities $f_{c_{\mathrm{vir}}}$ and $f_{R}$, was used the analytic fitting functions presented in Mo et al. (1998). For each galaxy, if $\psi$ is the SFR in units of $M_{\odot} / y r$ and if we assume for the SFR surface density profile $\dot{\Sigma}_{*}(R)=\dot{\Sigma}_{*, 0} \exp \left(-1.4 R / R_{\mathrm{d}}\right)$, the central SFR surface density is then given by

$\dot{\Sigma}_{*, 0}=\frac{\psi}{2 \pi\left(R_{\mathrm{d}} / 1.4\right)^{2}}$.

The gas surface density $\Sigma_{\text {gas }}$ is then determined by the K98 relation, and the gas mass $M_{\text {gas }}\left(\right.$ in $\left.M_{\odot}\right)$ is given by

$M_{\text {gas }}=\Sigma_{\text {gas }} \times 2 \pi R_{\mathrm{d}}^{2}$.

At this point we have a relation between $\mu$ and the stellar mass for each considered galaxy as shown in Fig. 2.

The aim of this work is to constrain the model parameters of simple models to reproduce the analytical fit (Eq. (15)) of the $\mathrm{MZ}$ relation. In our reference model we assume the value of $y_{\mathrm{O}}=0.01$ for the oxygen yield, a yield obtained by adopting the Salpeter IMF $\left(x=1.35\right.$ over the mass range $\left.0.1-100 M_{\odot}\right)$, the same one used for the inversion of the K98 law, and the stellar yields of Woosley \& Weaver (1995) for oxygen at solar metallicity. In the first part of our work, we explore the effect of a different choice of yields in the closed models. Given a stellar mass content $M_{*}$, we have an oxygen abundance value from the observed MZ relation, and then for each galaxy we have an estimate of the gas fraction $\mu$ using the procedure described above.

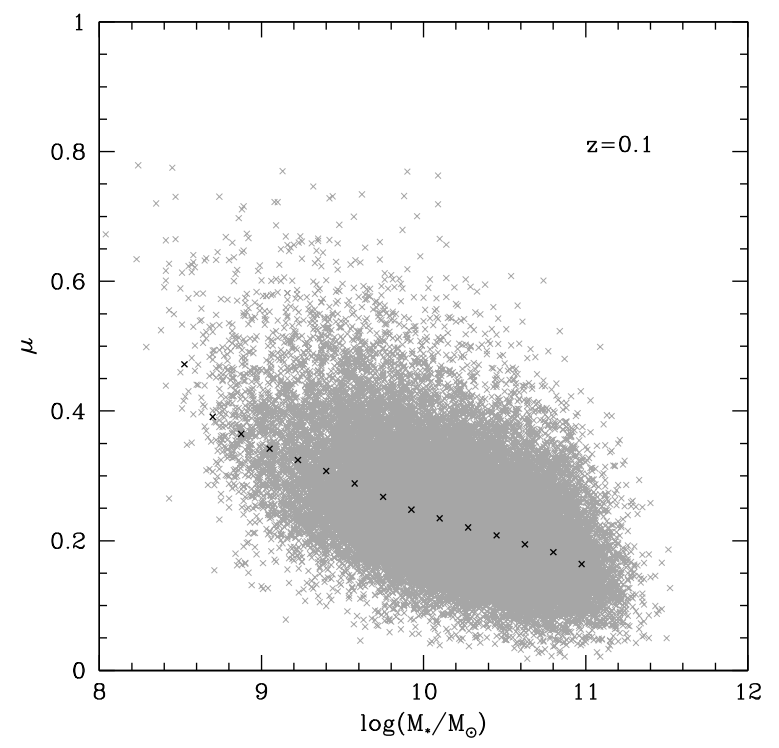

Fig. 2. The gas fraction $\mu$ as a function of the stellar galactic mass $M_{*}$. We divide the stellar masses in bins, and the average $\mu$ values for each bin are indicated by black crosses.

Therefore in closed models the only free parameter is the yield. We also show the effect of a non-constant yield, assuming is the IMF that varies and not the nucleosynthesis from galaxy to galaxy.

Concerning leaky box models, if we fix $Z$ and $\mu$ we can vary the wind and infall parameters, as well as the yield. We aim at testing the way in which the parameter space is constrained if we want to reproduce the observed $\mathrm{MZ}$ relation using simple-model solutions.

\section{Results}

\subsection{Closed box model results}

First of all we report our results for the closed model. In this case we can only vary the yield in the Eq. (3), because once $M_{*}$ is fixed, the $Z$ is given by the observational fit of Maiolino et al. (2008) and $\mu$ from the inversion of the Kennicutt law. In the Fig. 3 we report the simple-model solution compared to the observational average values and the standard deviation for the MZ relation. If we consider the simple model by adopting a constant oxygen effective yield of $y_{\mathrm{O}}=0.01$, observational data are clearly not reproduced. The predicted slope is too flat as shown in Fig. 3. Even if we consider a smaller yield, the problem is not solved: the MZ is simply translated at lower values with the same slope. The next step is to consider a variable yield as a function of the $M_{*}$. In the upper panel of Fig. 4 we report the yields per stellar generation as a function of the metallicity obtained to reproduce the MZ relation, whereas in the lower panel $y$ is as a function of stellar mass. The best fit yields spans in the range between 0.004 and 0.007 .

\subsection{Leaky box results}

In this section we show our results in the leaky box framework. We keep the oxygen yield fixed at $y_{0}=0.01$. First of all we present results for systems in which only outflows (Eq. (7)) and only infall (Eq. (9)) are included, separately. In the upper panel of Fig. 5 we report the best-fit $\Lambda$ and $\lambda$ parameters as a function of stellar mass in galaxies for reproducing the $\mathrm{MZ}$ relation. 
E. Spitoni et al.: The origin of the mass-metallicity relation: an analytical approach

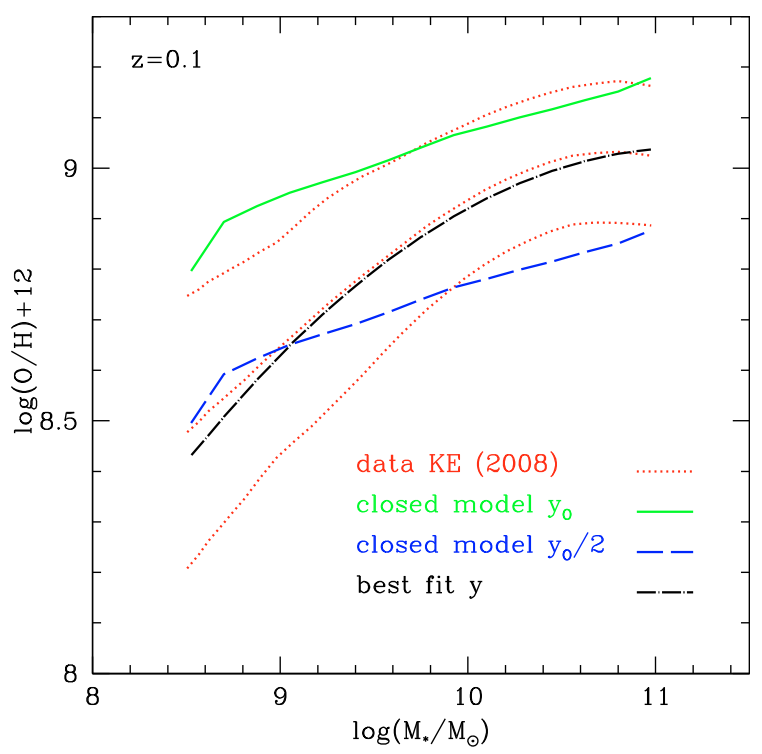

Fig. 3. The observed MZ relation and related standard deviation as indicated with the dotted red lines. With the green solid line we show our closed box model results assuming for the oxygen a yield per stellar generation of $y_{0}=0.01$, whereas the dashed blue line shows the model with $y_{\mathrm{O}}=0.005$. The model which best fits the data by using a variable yield is labeled with the short dashed dotted line.
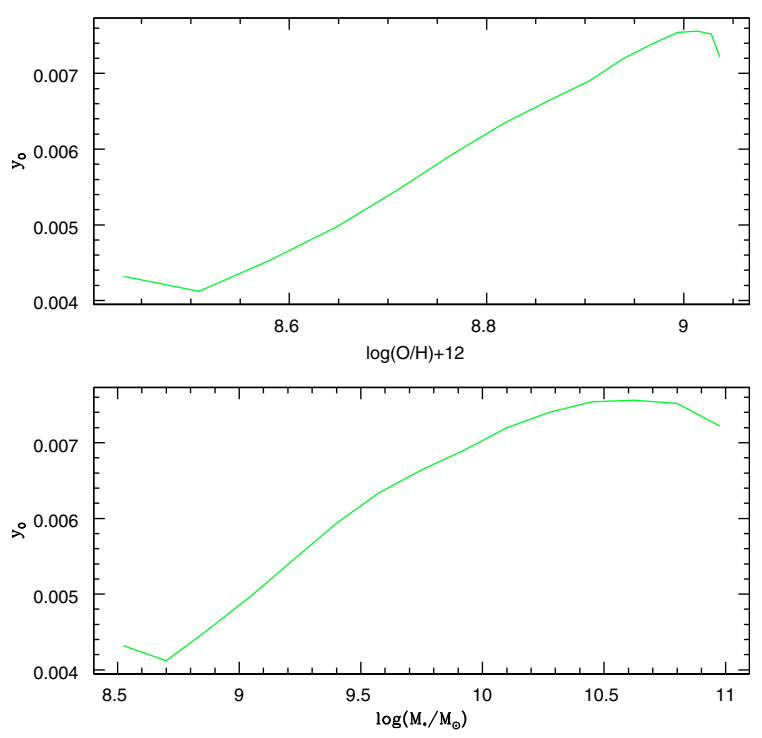

Fig. 4. In the lower panel we report the $y_{\mathrm{O}}$ yield values as functions of the galactic stellar mass $M_{*}$, those obtained to reproduce the MZ relation, while in the upper panel we show the same yields as functions of the derived $\mathrm{O}$ abundances.

Both the parameters, as expected, anti-correlate with the stellar mass in galaxies. For stellar masses lower than $10^{9.5}$, we have $\lambda \gg \Lambda$. If we look at the MZ relation plot obtained with the best fits for $\lambda$ and $\Lambda$ as reported in the lower panel of Fig. 5, we see that for $M_{*}<10^{9.5} M_{\odot}$ the model with only infall does not reproduce the observed MZ relation at all. The reason for this is the condition (10) holds for values of $\Lambda>1$, and this relation can be seen as a condition for $\Lambda$ values:

$\Lambda \leq \frac{1}{1-\mu}$
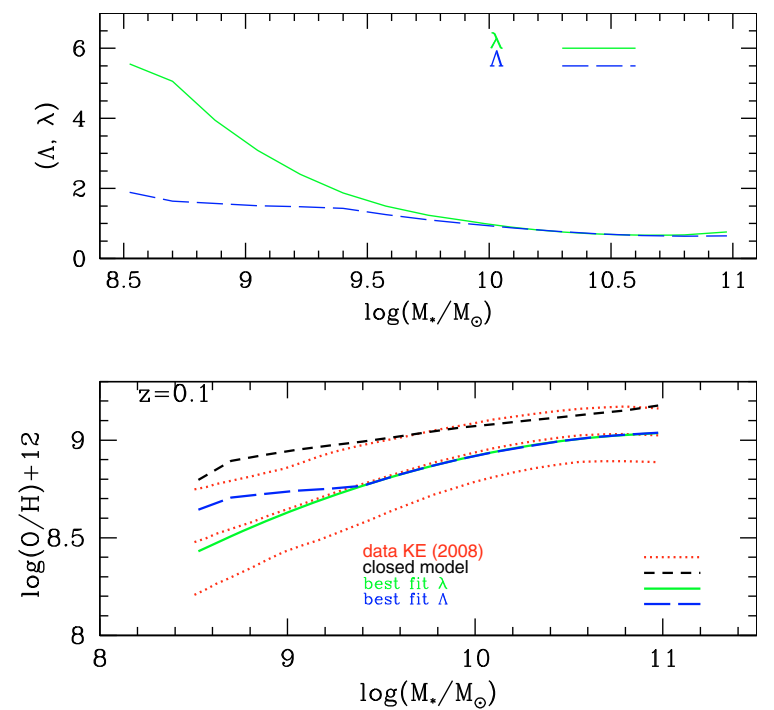

Fig. 5. Upper panel: the best $\Lambda$ and $\lambda$ values obtained for models with only infall and outflow, respectively, in order to reproduce the MZ relation. $\Lambda$ and $\lambda$ are given as functions of the galactic stellar mass $M_{*}$. Lower panel: the best fit to the observed MZ relation and related standard deviation are indicated with the dotted red lines. With the green solid line we show our best model results considering only outflow, while with the long dashed blue line are the best results for the model with only infall. The short dashed line indicates the results of the closed box model. In all models a value of $y_{\mathrm{O}}=0.01$ is adopted.

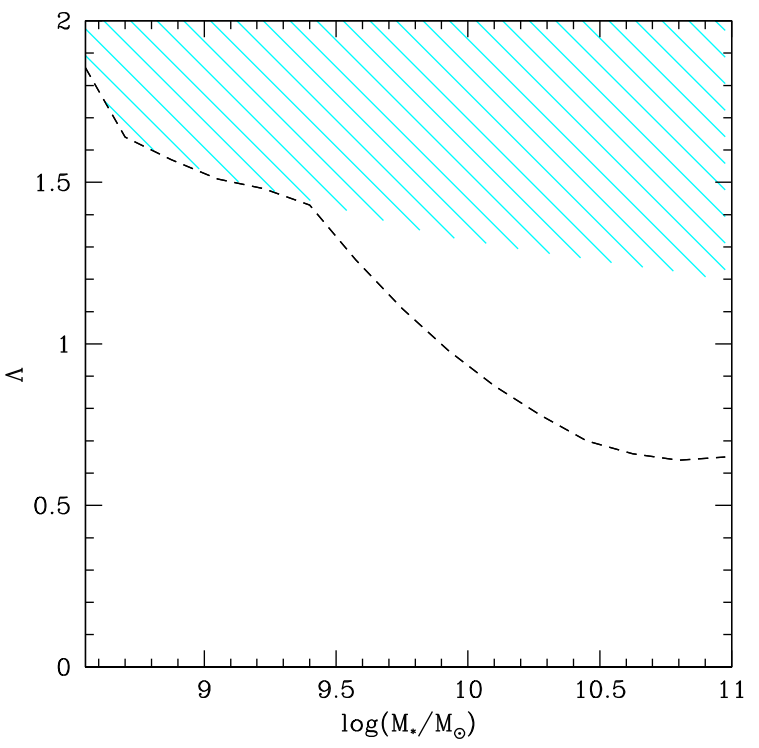

Fig. 6. Case with only infall: dashed area represents the forbidden $\Lambda$ values, the rest of the area represents the allowed values. With the short dashed line we draw the values of $\Lambda$ as a function of $\log \left(M_{*} / M_{\odot}\right)$ adopted in the MZ relation of Fig. 5.

In Fig. 6 there are the forbidden and allowed $\Lambda$ values. We also draw the values of $\Lambda$ as a function of $\log \left(M_{*} / M_{\odot}\right)$ relative to the predicted MZ relation of Fig. 5.

When $\log \left(M_{*} / M_{\odot}\right)=8.5$ and $\mu=0.47$, the $\Lambda$ values must be $<1.88$, whereas for $\log \left(M_{*} / M_{\odot}\right)=11.0$ and $\mu=0.16$ then $\Lambda<1.2$. Therefore, for the lower range of stellar masses considered, the derived $\Lambda$ does not allow the observed MZ relation to be reproduced. We conclude that the simple model solution concerning system with only infall varying with galactic stellarmass is unable to fit the MZ for small stellar mass galaxies even if we choose the best-fit $\Lambda$ values. 

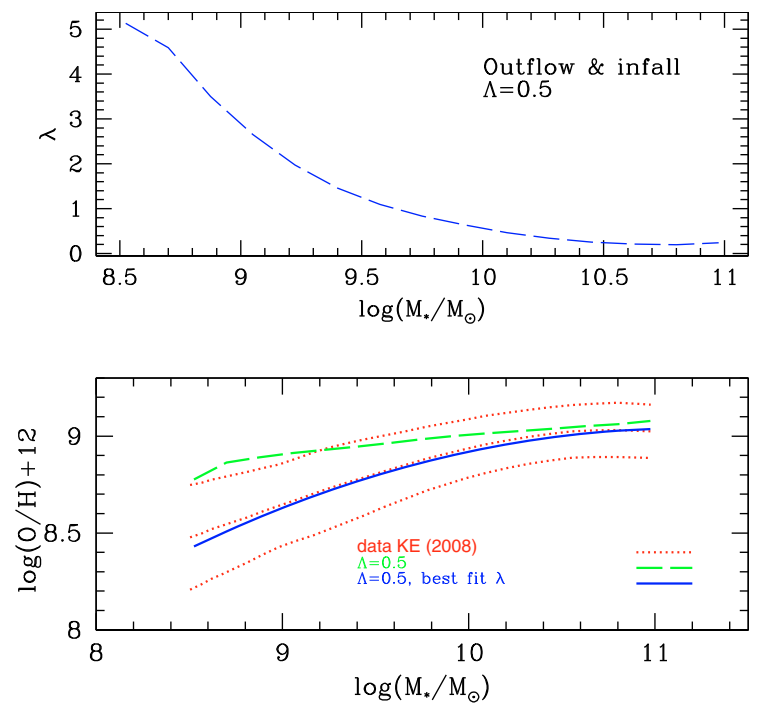

Fig. 7. Upper panel: the best $\lambda$ values that reproduce the MZ relation in the framework of the model with both infall and outflow. The infall parameter is fixed to be $\Lambda=0.5$. Lower panel: the best fit to the observed MZ relation and related standard deviation are indicated with the dotted red lines. With the blue solid line we show the results of the best model with both outflow and infall, obtained by fixing $\Lambda=0.5$ and varying $\lambda$. The dashed green line shows the model results with only infall with a constant $\Lambda=0.5$.

Concerning the solution of simple models with infall and outflow at the same time, we fix $\Lambda=0.5$ in Eq. (11), and we test which values of $\lambda$ must be assumed to reproduce the MZ relation. As reported in the upper panel of Fig. 7, the best-fit model for $\lambda$ anti-correlates with the stellar mass $M_{*}$.

In the lower panel we see that the MZ relation is reproduced by the best-fit model line. In the MZ plot we also include the result of the model only with the infall parameter $\Lambda$ equal to 0.5 . This model solution clearly does not reproduce the $\mathrm{MZ}$ relation. Therefore, a variable infall efficiency or a fixed one coupled with a variable outflow as a function of the galactic stellar mass is required.

We also analyze the case with a constant infall $\Lambda=1.1$ and a variable outflow as reported in Fig. 8. In the upper panel we see the behavior of the model with the best-fit $\lambda$ values. The choice of this particular value of $\Lambda$ is just arbitrary, but it does show the effect of a variable galactic wind in the presence of an infall with a fixed $\Lambda$ value, high enough to make the metallicity of large galaxies to decrease. We see that for $M_{*}$ higher than $10^{9.8} M_{\odot}$, $\lambda$ vanishes. The reason for this can be understood by analyzing the lower panel of Fig. 8. As in Fig. 7 we also report the solution with only the infall. We note that the infall with $\Lambda=1.1$ is too strong and flattens the MZ relation for high stellar mass systems. Therefore, if we couple this infall with an variable outflow, the best fit model is able to fit the MZ relation for systems with low stellar-mass values but certainly we cannot reproduce the part of the observed $\mathrm{MZ}$ where the infall was already too strong, namely for high galactic stellar masses. We find that the maximum value for the $\Lambda$ parameter, when varying $\lambda$ to reproduce the MZ relation, must be $\leq 0.7$.

The results from varying both the parameters are reported in Fig. 9. In the upper panel, the best-fit parameters as a function of $M_{*}$ are reported. These sets of best parameters lead to a difference in the MZ relation less than $10^{-5}$ dex between the best fit of Maiolino et al. (2008) and simple model solutions with this choice. In fact, in the lower panel of Fig. 9 comparing the
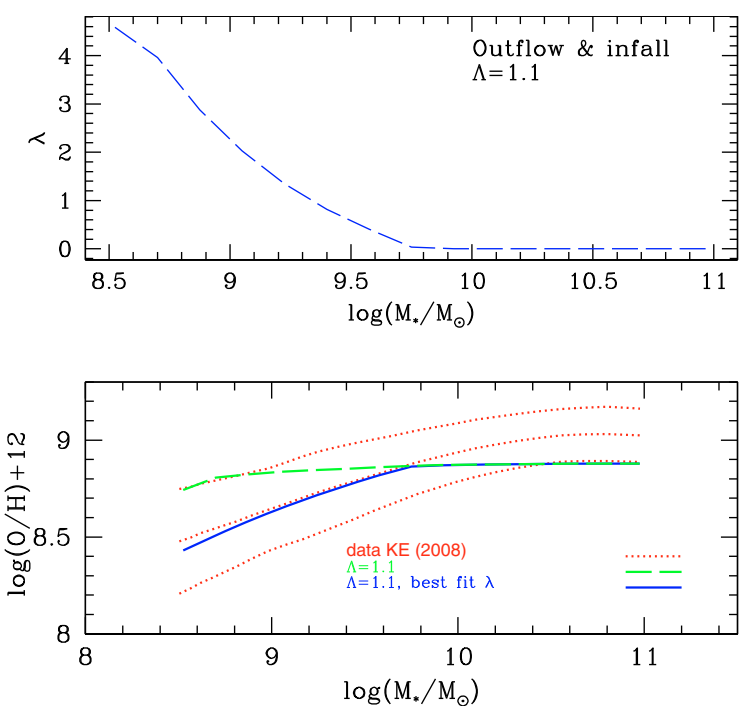

Fig. 8. Upper panel: the best $\lambda$ values for a model with both infall and outflow. $\lambda$ is given as a function of the galactic stellar mass $M_{*}$ with fixed $\Lambda=1.1$. Lower panel: the best fit to the observed MZ relation and related standard deviation are indicated with the dotted red lines. With the blue solid line, we show the best model results, obtained by considering both outflow in infall and by fixing $\Lambda=1.1$ while varying $\lambda$. The dashed green line shows the model solution with only infall with $\Lambda=1.1$
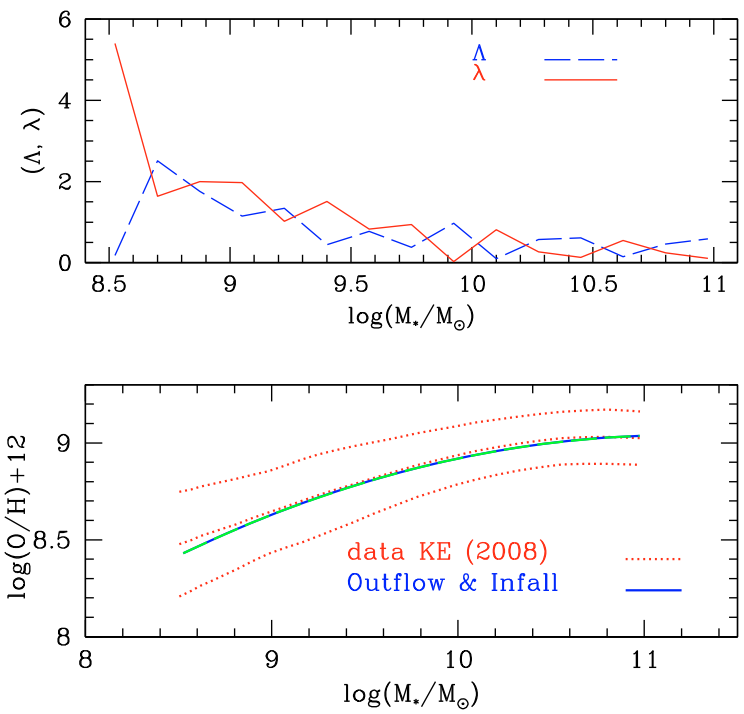

Fig. 9. Upper panel: the best-fit values for $\Lambda$ and $\lambda$ parameters in a model with infall and outflow, varying both the parameters at the same time. Lower panel: the best-fit to the observed MZ relation and related standard deviation are indicated with the dotted red lines. With the blue solid line we show the results of the best model adopting the $\Lambda$ and $\lambda$ values of the upper panel. The dashed green line shows the analytical fit of Maiolino et al. (2008).

Maiolino's fit and our best fit, we see that the two lines overlap perfectly. It is worth noting that in most of the mass bins (at least for $M_{*}<10^{9.5} M_{\odot}$ ) is $\lambda>\Lambda$.

The last part of our work is focused on simple model solutions with infall and differential winds. We presented in Sect. 2 the analytical solution found by Recchi et al. (2008). Considering also in this case a model with primordial infall, i.e. $Z_{A}=0$,we studied systems with 3 sets of parameters for $\Lambda$ and $\lambda:(\Lambda, \lambda)=(0.1,0.3) ;(0.2,0.6) ;(1,3)$. In the upper panel of Fig. 10 we show the variation in the possible $\beta$ values that 
E. Spitoni et al.: The origin of the mass-metallicity relation: an analytical approach
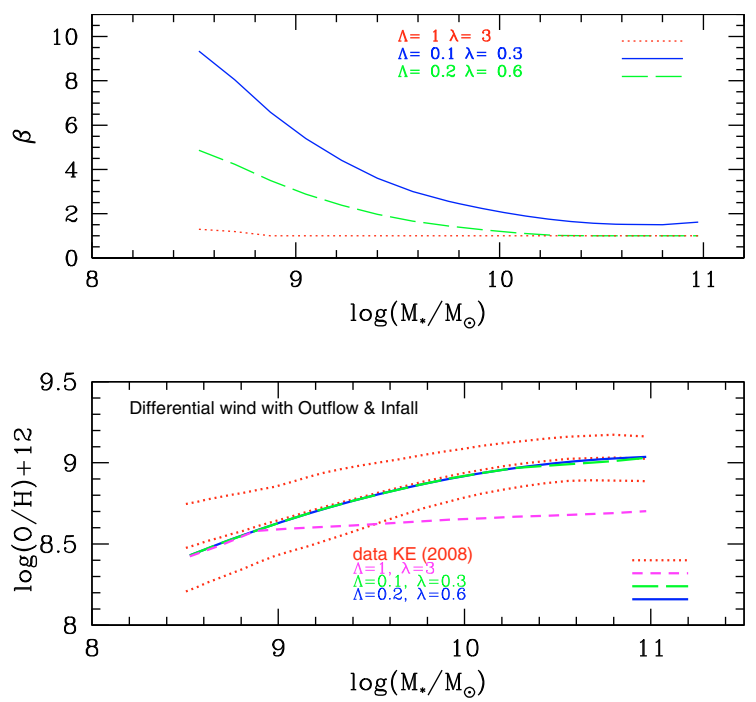

Fig. 10. Upper panel: the best values for $\beta$ in a model with both infall and outflow. The blue solid line refers to a system with $\Lambda=0.1$ and $\lambda=0.3$, the results for $\Lambda=0.2$ and $\lambda=0.6$ are indicated with the long dashed green line, and with the red short dashed line is shown the result with $\Lambda=1$ and $\lambda=3$. Lower panel: the best-fit to the observed MZ relation and related standard deviation (the dotted red lines) are shown. With the blue solid line we show the results of the best model obtained by varying the ejection efficiency $\beta$ and including also outflow and infall with $(\Lambda, \lambda)=(0.2,0.6)$. The cases with $(\Lambda, \lambda)=(0.1,0.3)$ and $(\Lambda, \lambda)=(1,3)$ are indicated with the green long dashed line and the magenta short dashed line, respectively.
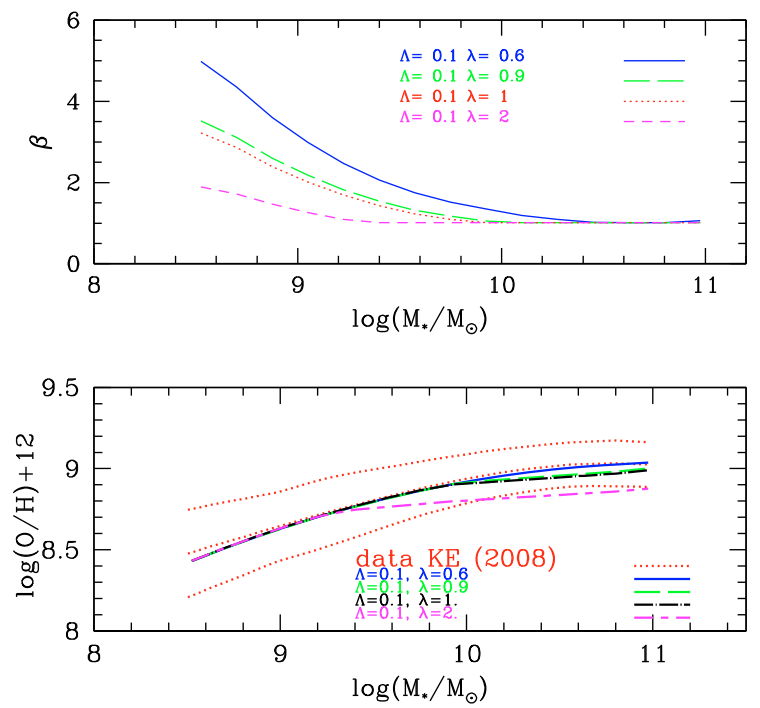

Fig. 11. Upper panel: the best values for $\beta$ in a model with both infall and outflow. We fix here the value of $\Lambda=0.1$ and vary $\lambda$. Lower panel: the best fit to the observed MZ relation and the related standard deviation are indicated with the dotted red lines. We fix the value of $\Lambda=0.1$. The other lines refer to the models in the upper panel.

can be considered in order to reproduce the MZ relation. As expected, for all models the best fit for $\beta$ anti-correlates with the galactic stellar mass, and the values of $\beta$ are higher for the first model $(\Lambda, \lambda)=(0.1,0.3)$. Both the first and the second model can reproduce the $\mathrm{MZ}$ relation. In the case with $(\Lambda, \lambda)=(1,3)$ instead, we are not able to reproduce the MZ relation, as shown in the lower panel of Fig. 10. Moreover, as shown in Fig. 11 we fix the infall parameter at the value $\Lambda=0.1$, and we find that the

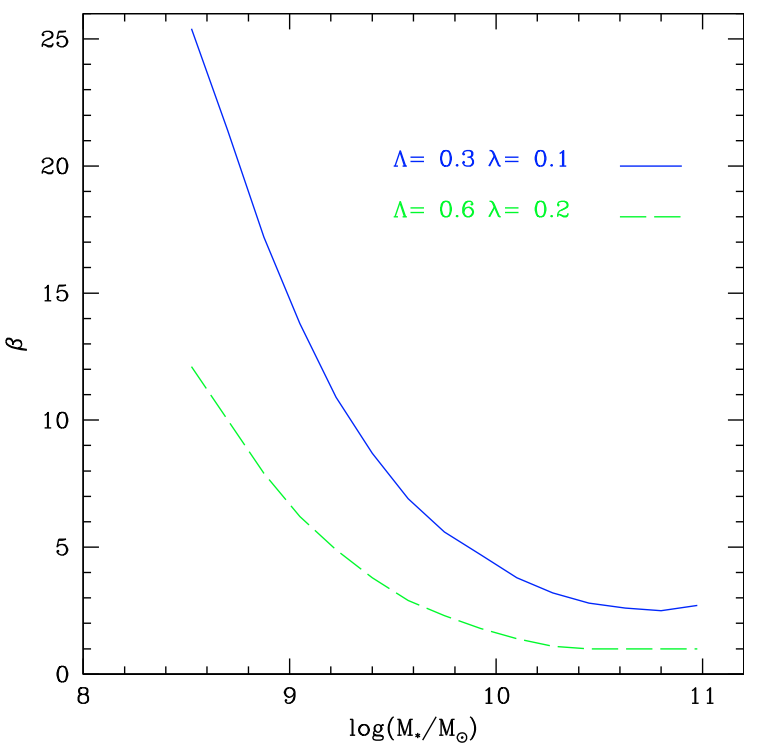

Fig. 12. The best-fit values for $\beta$ in a model with both infall and outflow. The blue solid line refers to a system with $\Lambda=0.1$ and $\lambda=0.3$, whereas the results for $\Lambda=0.2$ and $\lambda=0.6$ are indicated with the long dashed green line.

upper limit value for $\lambda$, in order to fit the MZ relation, is $\sim 0.6$ and in this case $\beta$ ranges between 1 and 5 .

We also study the case in which $\Lambda>\lambda$, with this set of parameters: $(\Lambda, \lambda)=(0.3,0.1) ;(0.6,0.2) ;(3,1)$. Here, the solution is defined only for values of $\mu$ such that

$\mu>\frac{\Lambda-\lambda-1}{\Lambda-\lambda}$,

as in the case of the model with outflow and infall. Using the set $(3,1), \mu$ must be greater than 0.5 . The observed average $\mu$ spans the range between 0.16 and 0.47 , then the $(3,1)$ set of parameters should be discarded. Both the first and the second models can reproduce the $\mathrm{MZ}$ relation, but the set $(0.3,0.1)$ requires values of $\beta$ that are too high and unrealistic in comparison with the wind parameter $\lambda$, as shown in Fig. 12, so it should also be rejected.

In Table 1 we summarize the permitted values of the parameters for reproducing the $\mathrm{MZ}$ relation for all the cases studied in this work.

\section{Conclusions}

In this paper we have explored different solutions for the MZ relation observed in SDSS galaxies. We adopted simple analytical models including infall, outflow, and differential outflow besides the classical closed-box case. All the solutions of the analytical models contain the yield per stellar generation plus several parameters describing the different physical processes considered. We started by deriving the gas mass for the sample of the studied galaxies with known stellar galactic masses and SFRs, by inverting the K98 law. Then, by means of these gas masses we derived the metallicity of each galaxy through the analytical models. At this point, we tried to vary the model parameters corresponding to the different physical processes to see whether the observed MZ could be reproduced. As it is well known, in order to obtain that metallicity increasing with galactic mass, the effective yield should decrease with decreasing mass. The effective yield can decrease because the outflow or infall becomes more important with decreasing mass or because the IMF becomes steeper. 
Table 1. Permitted values of the parameters for reproducing the MZ relation.

\begin{tabular}{|c|c|c|c|c|}
\hline Our Models & $\Lambda$ & $\lambda$ & $\beta$ & $y_{0}$ \\
\hline Only outflow & 1 & $0.67 \leq \lambda \leq 5.55$ & 1 & 0.01 \\
\hline Only infall & see Fig. 6 & 1 & / & 0.01 \\
\hline Outflow + infall (varying both $\Lambda$ and $\lambda$ ) & $0.10 \leq \Lambda \leq 2.51$ & $0.11 \leq \lambda \leq 5.40$ & / & 0.01 \\
\hline \multirow[t]{2}{*}{ Outflow + infall (fixed $\Lambda$ and varying $\lambda$ ) } & $0<\Lambda \leq 0.70$ & if $\Lambda=0.70 ; 0.16 \leq \lambda \leq 0.47$ & / & 0.01 \\
\hline & & if $\Lambda=0.01 ; 0.66 \leq \lambda \leq 5.54$ & / & 0.01 \\
\hline \multirow[t]{2}{*}{ Enriched Outflow + infall with $\Lambda<\lambda($ fixed $\Lambda=0.1)$} & 0.1 & $0.10<\lambda \leq 0.60$ & if $\lambda=0.60 ; 1.0 \leq \beta \leq 5.0$ & 0.01 \\
\hline & & & if $\lambda=0.11 ; 3.3 \leq \beta \leq 24.4$ & 0.01 \\
\hline Enriched Outflow + infall with $\Lambda>\lambda($ fixed $\Lambda=0.30$ and $\lambda=0.10)$ & 0.3 & 0.1 & $2.5 \leq \beta \leq 25.4$ & 0.01 \\
\hline Enriched Outflow + infall with $\Lambda>\lambda($ fixed $\Lambda=0.6$ and $\lambda=0.2)$ & 0.6 & 0.2 & $1.0 \leq \beta \leq 12.1$ & 0.01 \\
\hline Closed box + variable IMF & / & / & 1 & $0.004 \leq y_{0} \leq 0.007$ \\
\hline
\end{tabular}

Some of these cases have already been studied by other authors but never all together, and here we add a new case with differential outflow with wind intensity increasing with decreasing mass. By differential outflow we mean a galactic wind where metals are lost preferentially. Then, we tested whether the required parameter variations were realistic in order to choose among the different solutions.

Our conclusions can be summarized as follows.

- The observed local MZ relation can be perfectly reproduced under the assumption of a closed box and an effective yield varying from galaxy to galaxy. This means that either the nucleosynthesis is different or, more likely, that the IMF is different from galaxy to galaxy. The required yield variation is rather small and lies in the range 0.004-0.007, corresponding to a variation in the slope of the IMF (one-slope IMF defined in the mass range $0.1-100 M_{\odot}$ ) from $\sim 1.35$ to 1.5 going from more to less massive galaxies. However, the variation in the IMF always implies variations in other physical quantities besides the $\mathrm{O}$ abundance and therefore, before accepting this solution, it would be necessary to test other observables such as colors, the color-magnitude diagrams, and the $\mathrm{M} / \mathrm{L}$ ratios, if available. Besides that, if we change the IMF we should in principle also change the conversion factor from SFR to $\mu$ in the inversion of the Kennicutt law, which in our work has been calculated by adopting a Salpeter IMF in the mass range $0.1-100 M_{\odot}$ (see Sect. 3).

- The observed MZ relation can be reproduced very well by a constant yield but a variable efficiency of the outflow, increasing from more to less massive galaxies. This is not a new conclusion since it has been discussed before by several authors (Garnett 2000; Tremonti et al. 2004; Edmunds 2005). However, here we reached this conclusion by considering the most recent observational data. In particular, the wind parameter $\lambda$, which roughly represents the ratio between the wind rate and the SFR, should vary from 1 to 5.5, going from large to small galaxies, which is quite a reasonable range also supported by galactic wind observations (Martin et al. 2002). We have also found that outflows must play an important role especially for galaxies with stellar masses $<10^{10} M_{\odot}$. This is consistent with other results on the efficiency of outflows as a function of galactic mass, which show that dwarf galaxies must have ejected a fraction of their present baryonic mass greater than massive galaxies (e.g. Gibson \& Matteucci 1997; Calura et al. 2008).

- The local MZ relation cannot be reproduced by a variable infall efficiency, no wind, and constant IMF, so this solution should be rejected. This does not mean, however, that the infall is not important for the considered galaxies. Infall can be present, but it is not the cause of the MZ relation. Moreover, an infall rate increasing with decreasing galactic mass is not very easy to explain on a physical basis.

- Models with variable infall and outflow rates can, in fact, well reproduce the observed local $\mathrm{MZ}$ relation. In this case, the outflow rate is generally larger than the infall rate.

- Differential galactic winds, where metals are mostly lost from the galaxy, can also reproduce the observed MZ relation very well, provided that $\lambda>\Lambda$. In this case, the metal ejection efficiency $\beta$ is always greater in smaller galaxies. On the other hand, when $\Lambda>\lambda$ not all the values of $\Lambda$ and $\lambda$ are acceptable. Considering only the acceptable values for these parameters, we found that $\beta$ values, able to reproduce the MZ relation, are generally higher than in the case with $\Lambda<\lambda$.

The assumption of a larger $\beta$ in low-mass galaxies simply means that these galaxies are able to expel large fractions, relative to their total mass, of newly synthesized metals, but only tiny fractions of pristine ISM, in agreement with many hydrodynamical studies of star-forming dwarf galaxies (e.g. MacLow \& Ferrara 1999; D’Ercole \& Brighenti 1999; Recchi et al. 2001).

- We did not explore the case in which the efficiency of star formation increases with galactic mass, since the SFR does not appear in the solutions of the analytical models. Moreover, such a solution has already been explored in detail by Calura et al. (2009), who shows that this assumption reproduces the MZ relations both at low and high redshift. In addition, this in variation the star formation efficiency produces a downsizing effect in the star formation, which is often invoked to explain the properties of ellipticals (Pipino \& Matteucci 2004).

- In conclusion, on the basis of this paper and previous results of Calura et al.'s (2009), the most plausible solution is that the $\mathrm{MZ}$ relation is created by variable star formation efficiency from galaxy to galaxy coupled to galactic winds (preferentially metal-enhanced) becoming more and more important in low-mass galaxies. But as stressed by Edmunds (2005), explaining the mass-metallicity relation by a systematic increase in the star formation efficiency with galactic mass poses the problem of understanding the physical mechanism behind it. Several suggestions exist in the literature, but the real physical origin of this correlation is not known. A possibility is that the higher the pressure in the ISM, the higher the star formation efficiency, and the more massive galaxies have higher ISM pressure because of their deeper potential wells (see Elmegreen \& Efremov 1997; Harfst et al. 2006).

Acknowledgements. We thank the referee, M. Edmunds, for the enlightening suggestions. We acknowledge financial support from MIUR (Italian Ministry 
E. Spitoni et al.: The origin of the mass-metallicity relation: an analytical approach

of Research, contract PRIN2007 Prot.2007JJC53X-001) and from Italian Space Agency contract ASI-INAF I/016/07/0.

\section{References}

Barnes, J., \& Efstathiou, G. P. 1987, ApJ, 319, 575

Brooks, A. M., Governato, F., Booth, C. M., et al. 2007, ApJ, 655, L17

Bullock, J. S., Dekel, A., Kolatt, T. S., et al. 2001, ApJ, 555, 240

Calura, F., Jimenez, R., Panter, B., Matteucci, F., \& Heavens, A. F. 2008, ApJ, 682,252

Calura, F., Pipino, A., Chiappini, C., Matteucci, F., \& Maiolino, R. 2009, A\&A, 504, 373

Dalcanton, J. J. 2007, ApJ, 658,941

Dalcanton, J. J., Yoachim, P., \& Bernstein, R. A. 2004, ApJ, 608, 189

Dekel, A., \& Silk, J. 1986, ApJ, 303, 39

D'Ercole, A., \& Brighenti, F. 1999, MNRAS, 309, 941

De Lucia, G., Kauffman, G., \& White, S. D. M. 2004, MNRAS, 349, 1101

De Rossi, M. E., Tissera, P. B., \& Scannapieco, C. 2007, MNRAS, 374, 323

Edmunds, M. G. 1990, MNRAS, 246, 678

Edmunds, M. G. 2005, Astronomy \& Geophysics, 46, 4.12

Elmegreen, B. G., \& Efremov, Y. N. 1997, ApJ, 480, 235

Erb, D. K. 2008, ApJ, 674, 151

Erb, D. K., Steidel, C. C., Shapley, A. E., et al. 2006, ApJ, 646, 107

Finlator, K., \& Davé, R. 2008, MNRAS, 385, 2181

Garnett, D. T. 2004, MNRAS, 349, 491

Gibson, B. K., \& Matteucci, F. 1997, ApJ, 475, 47

Harfst, S., Theis, C., \& Hensler, G. 2006, A\&A, 449, 509

Hartwick, F. D. A. 1976, ApJ, 209, 418

Heavens, A. F., \& Peacock, J. 1988, MNRAS, 232, 339
Jimenez, R., Padoan, P., Matteucci, F., \& Heavens, A. F. 1998, MNRAS, 299, 123

Kennicutt, R. C. 1998, ApJ, 498, 541

Kewley, L. J., \& Dopita, M. A. 2002, ApJS, 142, 35

Kewley, L. J., \& Ellison, S. L. 2008, ApJ, 681, 1183

Kobayashi, C., Springel, V., \& White, S. D. M. 2007, MNRAS, 376, 1465

Köppen, J., Weidner, C., \& Kroupa, P. 2007, MNRAS, 375, 673

Larson, R. B. 1974, MNRAS, 169, 229

Larson, R. B. 1976, MNRAS, 176, 31

Lequeux, J., Peimbert, M., Rayo, J. F., Serrano, A., \& Torres-Peimbert, S. 1979, A\&A, 80, 155

Mac Low, M. M., \& Ferrara, A. 1999, ApJ, 513, 142

Maiolino, R., Nagao, T., Grazian, A., et al. 2008, A\&A, 488, 463

Martin, C. L., Kobulnicky, H. A., \& Heckman, T. M. 2002, ApJ, 574, 663

Matteucci, F. 1994, A\&A, 288, 57

Matteucci, F. 2001, The Chemical Evolution of the Galaxy, ASSL (Kluwer Academic Publisher)

Mo, H. J., Mao, S., \& White, S. D. M. 1998, MNRAS, 295, 319

Mouhcine, M., Gibson, B. K., Renda, A., \& Kawata, D. 2008, A\&A, 486, 711

Pagel, B. E. J., \& Patchett, B. E. 1975, MNRAS, 172, 13

Pipino, A., \& Matteucci, F. 2004, MNRAS, 347, 968

Recchi, S., Matteucci, F., \& D’Ercole, A. 2001, MNRAS, 322, 800

Recchi, S., Spitoni, E., Matteucci, F., \& Lanfranchi, G. A. 2008, A\&A, 489, 555 Silk, J. 2003, MNRAS, 343, 249

Somerville, R. S., Barden, M., Rix, H. W., et al. 2008, ApJ, 672, 776

Tassis, K., Kravtsov, A. V., \& Gnedin, N. Y. 2008, ApJ, 672, 888

Tinsley, B. M. 1980, Fund. Cosmic Phys., 5, 287

Tissera, P. B., De Rossi, M. E., \& Scannapieco, C. 2005, MNRAS, 364, L38

Tremonti, C. A., Heckman, T. M., Kauffmann, G., et al. 2004, ApJ, 613, 898

Twarog, B. 1980, ApJ, 242, 242

Woosley, S. E., \& Weaver, T. A. 1995, ApJS, 101, 181 